\title{
Culture Wars and Opinion Polarization: The Case of Abortion ${ }^{1}$
}

\author{
Ted Mouw \\ University of North Carolina
}

Michael E. Sobel

Columbia University

Recent observers have pointed to a growing polarization within the U.S. public over politicized moral issues-the so-called culture wars. DiMaggio, Evans, and Bryson studied trends over the past 25 years in American opinion on a number of critical social issues, finding little evidence of increased polarization; abortion is the primary exception. However, their conclusions are suspect because they treat ordinal or nominal scales as interval data. This article proposes new methods for studying polarization using ordinal data and uses these to model the National Election Study (NES) abortion item. Whereas the analysis of this item by DiMaggio et al. points to increasing polarization of abortion attitudes between 1972 and 1994, this article's analyses of these data offers little support for this conclusion and lends weight to their view that recent concerns over polarization are overstated.

\section{INTRODUCTION}

Is America wracked by "culture wars"-divisive debates in the media over issues such as abortion rights, affirmative action, homosexuality, public funding for the arts, and so on - that polarize public opinion into opposing viewpoints? According to proponents of the "culture wars hypothesis," the erosion of a common ground for reasoned ethical debate makes it difficult to resolve politicized moral issues and portends dan-

\footnotetext{
${ }^{1}$ An earlier version of this article was presented at the 1998 Chicago winter meetings of the methodology section of the American Sociological Association. We would like to thank Robert Kaufman, Yu Xie, Paul DiMaggio, and the AJS reviewers for comments and suggestions. Direct correspondence to Ted Mouw, Department of Sociology, CB 3210, 155 Hamilton Hall, University of North Carolina, Chapel Hill, North Carolina 27599-3210. E-mail: tedmouw@email.unc.edu
}

(C) 2001 by The University of Chicago. All rights reserved. 0002-9602/2001/10604-0002\$02.50 
American Journal of Sociology

gerous escalations in levels of social conflict (Hunter 1991, 1994; Bennet 1992; Gitlin 1995; Hochschild 1995). This same concern is echoed in the lay literature on contemporary politics (e.g., Shor 1986; Bolton 1992; Jacoby 1994; Bloom 1992; Gates 1992; Hartman 1991), as well as in the press. The Washington Post reported that between 1993 and 1996 there were approximately 1,500 articles in the media referring to contemporary American "culture wars." Apparently, the public also believes that Americans are culturally fragmented, with $86 \%$ of the respondents to a 1995 Newsweek poll agreeing that Americans share fewer common values now than in the past.

Others claim the concerns above are overblown, arguing that public opinion data indicate that the majority of people do not hold "extreme" views on divisive social issues (Brint 1992; Dillon 1996). For example, most national surveys about abortion show that the majority of Americans think abortion should neither be strictly legal or illegal in all circumstances (Blake and Del Pinal 1981; Gallup Poll News Service 1998). However, this does not address the basic question posed by the culture wars hypothesis: while public debate in America has always been contentious, is there something special about the contemporary "culture wars" that makes Americans more polarized now on important social issues than they were in the past? To address this hypothesis, it is not sufficient to measure polarization at a single point in time-we need to model trends in polarization over time.

In an important recent article, DiMaggio, Evans, and Bryson (1996, p. 693) argued that polarization as a state "refers to the extent to which opinions on an issue are opposed in relation to some theoretical maximum," while polarization as a process "refers to the increase in such opposition over time." To investigate polarization in the latter sense, they examine the change in variance and kurtosis of opinion items over time, with increasing variance and declining kurtosis taken to evidence polarization. Using time series of items from the General Social Survey (GSS) and National Election Study (NES), spanning the period 1972-94, DiMaggio et al. find no evidence for polarization of attitudes concerning crime and justice, gender, race, and sexual morality. They do find that attitudes toward abortion polarized over this period. However, their conclusions are suspect because they apply statistical methods appropriate for interval data to data that are nominal or ordinal. For example, to study trends in the binary GSS abortion items, they create a scale by summing the scores for each item and treating the resulting scale as interval (in general, scales created in this fashion are not even ordinal). To analyze the four-category ordinal NES abortion item, they score the re-

${ }^{2}$ Washington Post, February 23, 1997, final ed., p. C5. 
sponse categories $1,2,3,4$, and treat the resulting data as interval (see app. B, table B1). However, it is misleading to treat ordinal data as interval data because the scores assigned to the categories are arbitrary-only the relative ranking of the categories is known.

Our article makes several contributions. We develop appropriate methods for studying polarization in ordinal variables, using these to model trends in attitudes toward abortion and to ascertain whether or not polarization is increasing. We proceed as follows. First, we discuss the relevance of the abortion issue to the literature on culture wars and review previous work on trends in abortion attitudes. In the data and methods section, we describe the ordinal NES abortion item analyzed by DiMaggio et al. (1996) and several problems with their analysis, subsequently expositing (in context) the generalization of the cumulative probit model we developed to make inferences about trends in latent attitudes toward abortion. (A general discussion of the model and its implementation-in the Stata software package-is given in app. A.) We treat the observed NES item as an ordinal variable, making the usual type of assumption that responses to this item are the result of quantizing a normally distributed latent attitude, and we develop models that are consistent with this assumption. Using the models, we find no evidence for an increase in polarization between 1972 and 1980. While several other models offer some support for the view that polarization increased between 1980 and 1994, our best-fitting models, derived from theoretical considerations, do not support this view; a number of other considerations also suggest the superiority of these models over those that give evidence of polarization. Thus, as abortion is the exemplar issue in much of the culture wars literature, our findings suggest that the concerns expressed therein are overstated.

\section{SOCIAL CONFLICT OVER ABORTION RIGHTS: CULTURE WARS?}

"Culture wars" may be characterized as conflicts over issues that are rooted in nonnegotiable conceptions of cultural and moral order (Hunter 1991, 1996; Wuthnow 1996). According to Wuthnow and Hunter, these conflicts stem from a breakdown of the old denominational religious loyalties in America, with the traditional denominations splitting along a crosscutting conservative/liberal or orthodox/progressive divide that threatens the normative consensus (Davis and Robinson 1996). Hunter (1991) argues that the orthodox side stresses scriptural inerrancy and the belief that God is the ultimate arbiter of right and wrong, while progressives claim that morality is a human construct that is constantly evolving. Disagreement based upon fundamentally different moral frameworks 
American Journal of Sociology

is especially difficult to resolve given the lack of common ground between opposing viewpoints. Instead of a substantial number of moderates who can work toward compromise, the spectrum of opinion is increasingly polarized into irreconcilable viewpoints. Unable to negotiate between these competing moral views, debate thereby ceases to be an attempt to convince the opposition with reason and becomes instead a struggle for the power to impose a particular moral order. For these reasons, the new moral divisions in U.S. society create polarizing tendencies (Hunter 1991, p. 43). Furthermore, even if the majority of the population is "middle of the road," it is argued that people will adopt more extreme views over time (Nolan 1996). According to Hunter (1991), this sort of discord is especially dangerous because it is antidemocratic-each side's position is seen as a fundamental right that transcends the democratic process. He argues that the metaphor "culture wars" should be taken literally: "Violence? The suggestion that violence can occur is not made lightly: culture wars always precede shooting wars. It is culture, after all, that justifies the use of violence. We are truly in the midst of a culture war of great social and historical significance" (1993, p. 30).

Moreover, the divisions between competing moral visions are so deep "there is no resolution to the culture war in America" (Hunter 1996, p. 253). If culture wars lead to increasing opinion polarization, then the decline in the proportion of people with moderate views makes it difficult to resolve politicized moral issues and may increase the likelihood that advocacy groups will resort to violence to impose their views.

The dispute over abortion rights appears to epitomize the foregoing arguments (Hunter 1994), as witnessed by a number of recent books whose titles call attention to the presumed irreconcilable viewpoints of the parties to this conflict: Abortion: The Clash of Absolutes (Tribe 1992), No Neutral Ground: Abortion Politics in the Age of Absolutes (O'Connor 1996), The Abortion Dilemma (Claire 1995), and Between Two Absolutes: Public Opinion and the Politics of Abortion (Cook, Jelen, and Wilcox 1992). In an ethnographic study of abortion activists, Luker $(1984$, p. 2) argues that pro-choice activist women "share almost no common premises and very little common language" with antiabortion activist women; in particular, the worldviews and conceptions of motherhood held by the two different groups of women are antithetical. Hunter (1994) argues that activist organizations further contribute to opinion polarization by conducting "symbolic warfare" with their opponents. According to him, activists on both sides of the abortion dispute attempt to coerce consent by relying on rhetorical distortions and emotional appeals, with the modern media exacerbating matters by encouraging superficial and confrontational discourse. Thus, the debate over abortion is reduced to a struggle over inflammatory images that limits the possibilities for compromise: the coat 
hanger for pro-choice groups and grisly pictures of aborted fetuses for pro-life groups. A pro-life activist interviewed by Hunter (1994, p. 67) states, "This is a visual war we're fighting here [because of] the 30-second sound-bite society we live in." Hunter concludes that the outcome of this sort of discourse is a "thinning out" of the middle ground, reducing the chances for democratic compromise.

Others do not view the abortion dispute as a clash between irreconcilable views. Ginsburg's (1989) study suggests that although pro-life and pro-choice activists come to hold different opinions on abortion, there are also significant commonalities in their views, with both types of activists emphasizing the importance of nurturance, for example. Dillon (1996) argues that the public opinion literature indicates that Americans have complex and nuanced views about abortion that are not readily understood using a simple orthodox/progressive dichotomy. She also argues, from her work on the language of the abortion debate, that the level of rhetorical complexity featured here is similar to the level encountered in political discussions of other issues. According to her, activists on both sides of the fence use arguments that attempt to engage those with opposing views, as opposed to summarily dismissing the contrary view out of hand. If this is the case, then the abortion dispute, while heated, does not differ from other forms of reasoned democratic discourse, implying that the concerns of writers on "culture wars" (at least on the abortion issue) are misdirected.

Nonetheless, Hunter's concern that increasing polarization portends outright civil violence should not be taken lightly. As table 1 shows, abortion violence and protest appear to have escalated in the first half of the 1990s, culminating in the murder of five abortion providers in 1993-94. These acts of violence and social protest, widely reported in the media, constitute the public face of the conflict over abortion and provide prima facie evidence for the concerns of Hunter and others who worry that the divide in U.S. society does not permit a peaceful resolution. However, some caution is in order. First, the level of violence associated with the abortion issue seems "small," perhaps reflecting the actions of isolated individuals or fringe groups. Second, much of the increase in abortion protest appears to be picketing, and this type of activity cannot be viewed as antidemocratic. Thus, to use the type of evidence in table 1 to suggest that polarization in the U.S. public is spiraling out of control, as some sociologists and journalists have done, seems unwarranted. Further, even were the prima facie evidence stronger, it has been argued (Ginsburg 1998; Risen and Thomas 1998) that in the wake of this violence both pro-life and pro-choice groups adopted more moderate positions in an attempt to distance themselves from extremists. Therefore, it is conceivable that recent violence will generate a decline in polarization. Finally, and most 
TABLE 1

InCidents Against Abortion Providers, I977-94

\begin{tabular}{|c|c|c|c|c|c|c|c|c|c|c|c|c|}
\hline & $1977-83$ & 1984 & 1985 & 1986 & 1987 & 1988 & 1989 & 1990 & 1991 & 1992 & 1993 & 1994 \\
\hline \multicolumn{13}{|l|}{ Violence: } \\
\hline Murder & 0 & 0 & 0 & 0 & 0 & 0 & 0 & 0 & 0 & 0 & 1 & 4 \\
\hline Attempted murder ... & 0 & 0 & 0 & 0 & 0 & 0 & 0 & 0 & 2 & 0 & 1 & 8 \\
\hline Bombing $\ldots \ldots \ldots \ldots$ & 8 & 18 & 4 & 2 & 0 & 0 & 2 & 0 & 1 & 1 & 1 & 3 \\
\hline Arson $\ldots \ldots \ldots \ldots \ldots$ & 13 & 6 & 8 & 7 & 4 & 4 & 6 & 4 & 10 & 16 & 9 & 6 \\
\hline \multicolumn{13}{|l|}{ Protest: } \\
\hline Picketing $\ldots \ldots \ldots \ldots \ldots$ & 107 & 160 & 139 & 141 & 77 & 151 & 72 & 45 & 292 & 2,898 & 2,279 & 1,407 \\
\hline Clinic blockades ..... & 0 & 0 & 0 & 0 & 2 & 182 & 201 & 34 & 41 & 83 & 66 & 25 \\
\hline Blockade arrests ..... & 0 & 0 & 0 & 0 & 290 & 11,732 & 12,358 & 1,363 & 3,885 & 2,580 & 1,236 & 217 \\
\hline
\end{tabular}




\section{Culture Wars}

important, no matter how tempting it is to do so, it is simply incorrect to infer the existence of increasing opinion polarization from the very outcomes such polarization is held to predict; social psychologists interested in the relationship between attitudes and behavior learned a similar lesson many years ago.

In short, it seems clear that in order to ascertain whether or not opinions are becoming more polarized, as argued by Hunter and other writers who speak of "culture wars," it is necessary to directly examine opinion data. Using this type of evidence, Brint (1992) and Dillon (1996) argued that the proportion of persons holding extreme views on the abortion issue is not large enough to evidence polarization. However, there are several problems with these conclusions. Most important, these authors do not examine time trends, as would be required to ascertain whether or not levels of polarization are increasing. Second, even if interest resided in polarization at a single point in time, the definition of an extreme response is subjective, and these authors offer no criteria for assessing the extent of polarization. Having concluded that it is necessary to examine trends in abortion attitudes to assess the validity of the culture wars hypothesis, we now examine previous work on temporal trends in abortion attitudes.

\section{TRENDS IN ABORTION ATTITUDES}

Gauging public opinion about abortion is difficult given the complexity of the moral and legal issues involved; in addition, responses to opinion items depend on question wording and order (Jaffe, Lindheim, and Lee 1981; Adamek 1994; Bumpass 1997). Nevertheless, several findings emerge consistently from the literature. First, at all points in time and on all major surveys, a substantial proportion of the population does not think that abortion should be strictly legal or illegal in all circumstances (see Cook et al. 1992; Hunter 1994). According to a 1998 Gallup poll, this percentage was $54 \%$ in $1975,48 \%$ in 1992 , and again $58 \%$ in $1998 .^{3}$ In this sense, it seems true that a large proportion of the population holds "middle of the road" views about abortion. Second, support for abortion varies widely according to the specific circumstances of the abortion. A large majority of individuals support abortion when the life of the mother is in danger or in the case of rape or incest. In contrast, there is considerably less support for abortion for social reasons, such as not being able to afford the child (Gallup Poll News Service 1979; Granberg and Gran-

${ }^{3}$ These data were mentioned in an article entitled "Fluctuations, but No Major Change in Views on Abortion" that was posted at CNN Interactive (http://www.cnn.com/US/ 9801/20/abortion.poll/index.html) in 1998. See also Gallup Poll News Service (1998) and Hall (1998). 
American Journal of Sociology

berg 1980; Cook et al. 1992). Third, the level of support for abortion has been fairly stable since the Roe v. Wade decision. While the level of support increased substantially from the early 1960s to the early 1970s, there was little change throughout the rest of the 1970s and a slight decline in the early 1980s (de Boer 1977; Glenn 1987; Gallup Poll News Service 1979; Granberg and Granberg 1980; Gillespie, Vergert, and Kingma 1987; Page and Shapiro 1992). By 1985, the net result was that the level of public support for abortion in the 1972 and 1985 GSS data is almost identical (Glenn 1987). Recent opinion data show some fluctuation in the support for abortion. According to Gallup survey data, between 1975 and 1998, the percentage of people who thought that abortion should be legal in all circumstances rose from $21 \%$ in 1975 to $29 \%$ in 1989 and $34 \%$ in 1992, but has since declined to 23\% in 1998 (Gallup Poll News Service 1998). The remarkable thing about this trend is that, despite these fluctuations, support for legal abortion is about the same as it was 20 years ago.

However, as Hunter (1994) and DiMaggio et al. (1996) note, aggregate measures of support for a particular issue - that is, the "average" opinion-fail to measure attitude polarization. To see this, simply imagine the case where the "average opinion" is temporally stable, with individuals on both sides of the center moving steadily toward the extremes. While the average opinion is unchanged, the variance is increasing; substantively, increased variability may make compromise between opposing views more difficult to obtain.

To address the issue of opinion polarization in abortion attitudes, DiMaggio et al. (1996) used two sources, the NES and the GSS. The NES is a biennial survey in which respondents were asked, beginning in 1972, to express their opinion about abortion on a four-point ordinal scale. The abortion item was not asked in 1974, but was asked in 1976, $1978, \ldots$, 1994. DiMaggio et al. scored the categories 1-4 and treated the resulting data as interval data, calculating (at each time point) the sample mean, variance, and kurtosis of the item responses; they then regressed the series of means, variances, and kurtoses against time. In 1980, the NES response codes were changed, but a subset of 1,320 of the respondents in that year were asked to respond using both the old and new response codes; for this year, they used only these respondents, defining the respondent's score as the average of the responses to the old and new items. Their analysis suggests that, over the study period, the variability in opinions increased and the kurtosis decreased, evidencing increasing polarization.

There are two major problems with DiMaggio et al.'s analysis of the NES data. First, because the NES item is ordinal, it is not appropriate to calculate means, variances, and the kurtosis of the item. Second, DiMaggio et al. (1996) pieced the NES data into a single series combining 
the old NES item (1972-78), the average of the old and new items (1980), and the new NES item (1982-94). We discuss these problems further in the next section and present appropriate statistical methods for assessing polarization in ordinal opinion items.

Using six binary items on abortion from the GSS, DiMaggio et al. also constructed a time series (with 14 points) spanning 1977-94. Each item was initially scored 1 or 2 , and the item scores were added, resulting in a scale value for each respondent. The resulting scale was treated as interval data, and the data were analyzed as above. According to the authors, opinion was already sharply divided at the onset of the series in 1977 , and polarization increased over the study period, as evidenced by increasing dispersion and declining kurtosis, with the decrease in kurtosis leveling off by the mid-1980s. This analysis is also problematic, as scales constructed in such a fashion need not even be ordinal, and even if the items are "unidimensional," that does not mean that a scale constructed in the manner above is interval. To analyze these data properly, it would be necessary either to examine the trends in each item separately or to construct a probabilistic model of the response process with an underlying latent attitude toward abortion.

Because the methods we present for analyzing polarization using an ordinal item are not necessarily appropriate for analyzing the GSS items, these data are not analyzed in this article. Although we do not accept the conclusions DiMaggio et al. reached using these data, the reader should note that it is possible that the GSS data, were they are analyzed properly, might still suggest that attitudes toward abortion polarized. We return to that issue in the discussion.

\section{DATA AND METHODS}

The NES is conducted biennially by the Institute for Social Research at the University of Michigan. The NES sample that we use is a nationally representative probability sample of all households in the United States. In 1992 and 1994, some of the NES respondents are panel respondents in ongoing longitudinal studies. For each year, the nonpanel respondents constitute a nationally representative sample, but the panel respondents do not; thus, only the nonpanel respondents are used in the analysis. Table 2 displays the the time series of data for both old and new questions.

Comparison of the response codes in table B1 reveals that the new item emphasizes the legality of abortion, while the old one does not, suggesting the two items do not measure the same latent attitude toward abortion. If this is the case, it was inappropriate for DiMaggio et al. to combine the two items to form a single series and then examine this series for 
American Journal of Sociology

TABLE 2

Responses to Abortion Questionnaire

\begin{tabular}{|c|c|c|c|c|c|c|}
\hline Year & $\begin{array}{c}\text { Never } \\
\text { Legal }\end{array}$ & $\begin{array}{l}\text { Life in } \\
\text { Danger }\end{array}$ & $\begin{array}{c}\text { Clearly } \\
\text { Established }\end{array}$ & $\begin{array}{c}\text { Always } \\
\text { Legal }\end{array}$ & Total & $N$ \\
\hline \multicolumn{7}{|l|}{ Old: } \\
\hline $1972 \ldots$ & 11.2 & 46.9 & 17.3 & 24.5 & 100 & 2,619 \\
\hline $1976 \ldots$ & 11.3 & 45.7 & 16.3 & 26.8 & 100 & 1,822 \\
\hline $1978 \ldots$ & 10.9 & 44.6 & 16.8 & 27.6 & 100 & 2,187 \\
\hline $1980 \ldots$ & 9.8 & 44.7 & 18.0 & 27.5 & 100 & 1,362 \\
\hline \multicolumn{7}{|l|}{ New: } \\
\hline $1980 \ldots$ & 11.5 & 32.7 & 19.0 & 36.8 & 100 & 1,547 \\
\hline $1982 \ldots$ & 13.3 & 30.8 & 19.6 & 36.4 & 100 & 1,359 \\
\hline $1984 \ldots$ & 13.3 & 30.3 & 20.0 & 36.4 & 100 & 2,171 \\
\hline $1986 \ldots$ & 13.4 & 29.0 & 18.4 & 39.3 & 100 & 2,120 \\
\hline $1988 \ldots$ & 12.7 & 33.2 & 18.5 & 35.6 & 100 & 1,996 \\
\hline $1990 \ldots$ & 12.2 & 33.2 & 14.4 & 40.2 & 100 & 1,932 \\
\hline $1992 \ldots$ & 10.8 & 26.2 & 13.8 & 49.2 & 100 & 1,087 \\
\hline $1994 \ldots$ & 12.6 & 30.8 & 14.7 & 42.0 & 100 & 994 \\
\hline
\end{tabular}

NotE. - Data are from the National Election Study. For 1992-94, sample excludes panel study members. See table B1 for a description of the categories.

trends. To address this important issue further, we use the 1,320 NES respondents who answered both the old and new abortion items in 1980. Table 3 contains the percentages, means, and variances for both sets of questions. Table 4 contains the cross-classification of responses to both sets of questions. If these items measure the same latent attitude and the relationship between the latent attitude and observed responses is the same for both items, the marginal distributions (see table 3) of the two items will be identical. If the two marginal distributions are not identical, either (1) the two items do not measure the same latent variable, in which case it is not appropriate to combine the years 1972-78 with the years 1980-94, or (2) the relationship between the latent attitude and the observed response is not identical for the two items, in which case assigning identical sets of scores to the two items and examining trends over the full period 1972-94 almost certainly confounds the item change in 1980 with any possible true attitudinal change. Inspection of table 3 suggests that the 1980-94 question elicits a lower level of response in category 2 and a higher level of response in category 4; further, the null hypothesis— that the two marginal distributions are identical—is rejected. ${ }^{4}$ There-

${ }^{4}$ To ascertain whether or not the distributions of the old and new NES items are
identical, we test for marginal homogeneity in the cross-classifications of old by new
responses for the 1,320 respondents in 1980 who answered both itmes. The data are
given in table 4 . Because the log-linear model of quasi-symmetry $(Q S)$ fits these data
adequately $\left(L^{2}[Q S]=7.21 ; d f=3 ; P=.07\right)$, where $L^{2}$ denotes the likelihood ratio $\chi^{2}$ 


\section{Culture Wars}

TABLE 3

Old and New Abortion Questions in i980, Percentages, Means, and Variances

\begin{tabular}{lcc}
\hline \hline Question/Category & Old & New \\
\hline $1 \ldots \ldots \ldots \ldots \ldots \ldots \ldots$ & 9.9 & 12.0 \\
$2 \ldots \ldots \ldots \ldots \ldots \ldots \ldots$ & 44.2 & 32.1 \\
$3 \ldots \ldots \ldots \ldots \ldots \ldots \ldots$ & 18.2 & 18.7 \\
$4 \ldots \ldots \ldots \ldots \ldots \ldots$ & 27.7 & 37.2 \\
Total $\ldots \ldots \ldots$. & 100.0 & 100.0 \\
Mean $\ldots \ldots \ldots \ldots \ldots$ & 2.64 & 2.81 \\
Variance $\ldots \ldots \ldots \ldots$ & .983 & 1.14 \\
\hline
\end{tabular}

Note. $-N=1,320$.

fore, the two items should not be combined into a single time series, as was the case in DiMaggio et al. ${ }^{5}$

To understand the effect of the change in question wording on the conclusions drawn by DiMaggio et al., we need to compare their analysis (described previously) with an analysis that adjusts the series of means, variances, and kurtoses for this change. To perform these adjustments, we (see table 3), used the 1980 data on the 1,320 respondents who answered both the old and new NES items, treating these data as interval data, as was done in DiMaggio et al. (1996). The means of the old and new items are, respectively, 2.638 and 2.811, which yields an estimate of the effect (on the mean) due to the change in wording as $2.811-$ $2.638=.173$; we therefore added .173 to the means for the pre-1980 data. For the data from 1980-94, we used the mean calculated from the new item. The variance series and the kurtosis series were adjusted similarly, using .983 for the variance of the old item and 1.140 for the variance of the new item, and -1.198 for the kurtosis of the old item and -1.319 for the kurtosis of the new item.

Without adjusting the data for changes due to wording, DiMaggio et al. (p. 716) regressed their series of means, variances, and kurtoses on time, leading them to conclude that, between 1972-94, attitudes toward abortion become more liberal, but also more polarized, as evidenced by increasing variability and decreasing kurtosis. Using the adjusted mean series, the estimated slope is .008 ( $\mathrm{SE}=.003 ; P=.011)$, slightly smaller than the estimated slope in DiMaggio et al.; For the variance series, the

statistic and $P$ denotes the $P$-value (based on $L^{2}$ ), marginal homogeneity is equivalent to symmetry $(S)$ (Agresti 1990, p. 390). therefore, the null hypothesis may be tested using the conditional likelihood ratio test $\left(L^{2}[S]-L^{2}[Q S]=133.69-7.21=125.48\right.$; $d f=3 ; P<.001)$; clearly, this leads to rejection at any conventional level of significance.

${ }^{5}$ One could overcome this problem if plausible adjustments were made to compensate for the effect of the change in wording. 
American Journal of Sociology

TABLE 4

Cross-Classification of Responses to the Old and New Abortion Questions, I980

\begin{tabular}{lrrrrr}
\hline \hline & \multicolumn{3}{c}{ New Question/Category } & \\
\cline { 2 - 4 } Old Question/Category & \multicolumn{1}{c}{1} & \multicolumn{1}{c}{2} & \multicolumn{1}{c}{3} & \multicolumn{1}{c}{ Total } \\
\hline $1 \ldots \ldots \ldots \ldots \ldots \ldots \ldots \ldots \ldots \ldots$ & 75 & 40 & 7 & 8 & 130 \\
$2 \ldots \ldots \ldots \ldots \ldots \ldots \ldots \ldots \ldots$ & 73 & 334 & 120 & 57 & 584 \\
$3 \ldots \ldots \ldots \ldots \ldots \ldots \ldots \ldots \ldots \ldots$ & 7 & 36 & 90 & 107 & 240 \\
$4 \ldots \ldots \ldots \ldots \ldots \ldots \ldots \ldots \ldots$ & 3 & 14 & 30 & 319 & 366 \\
Total $\ldots \ldots \ldots \ldots \ldots \ldots \ldots$ & 158 & 424 & 247 & 491 & 1,320 \\
\hline
\end{tabular}

NotE.-Source: National Election Study, 1980.

estimated slope is .003 ( $\mathrm{SE}=.0009 ; P=.009)$; although the estimated slope is now substantially smaller than that reported by DiMaggio et al., note that it is still significantly different from zero at the .05 level, indicative of increasing polarization. For the kurtosis, the estimate is -.004 ( $\mathrm{SE}=.003 ; P=.163)$; in DiMaggio et al., the slope is -.013 , significantly different from zero at the .05 level.

Our calculations suggest that when the ordinal NES item is adjusted for the change in item wording, but still inappropriately treated as interval data, there is still evidence (although not as strong as before) of increasing polarization. Similarly, our calculations suggest that attitudes became more liberal, but not to the extent suggested by DiMaggio et al. However, these calculations do not bear on the implicit assumption that the old and new NES items measure the same attitude.

Because the old NES item makes no reference to the legality of abortion-and therefore seems to tap personal beliefs, while the new NES item refers to legality and therefore seems to tap something other than purely personal beliefs - we do not believe it is reasonable to think the old and new items measure the same attitude. Therefore, in this article, we model the old NES abortion item using the data from 1972-78 and the respondents who answered the old item in 1980, and we separately model the new NES abortion item using the data from 1980-94. We ask whether polarization increased between 1972 and 1980 (using the old item) and whether or not polarization increased between 1980 and 1994 (using the new item).

The Model

We now discuss a model for ordinal dependent variables that allows us to more appropriately assess whether or not attitudes toward abortion have polarized in the past 25 years. The model we use to analyze the 


\section{Culture Wars}

data in table 2 is a cumulative probit model with heteroscedasticity and variable cutpoints. A general discussion of the model and its estimation is given in appendix A; here we exposit the model within the context of a four-point ordinal scale. In the usual cumulative probit model for an ordinal scale $Y$ with 4 categories, a latent variable indexing the unobserved attitude of respondent $i$ at time $t$ (for convenience of exposition, we index the time points $t=1, \ldots, T$ ) is postulated to follow a regression model:

$$
Y_{i t}^{*}=\mu_{t}+\varepsilon_{i t},
$$

where $\mu_{t}$ is the mean response for time $t$, and $\varepsilon_{i t}$ is a normally distributed error with mean zero and variance $\sigma^{2}$. The relationship between $Y_{i t}^{*}$ and the observed variable $Y_{i t}^{*}$ (the response on the four-point scale) is assumed to be of the form $Y_{i t}=1$ if $Y_{i t}^{*} \leq \tau_{1}, Y_{\text {it }}=2$ if $\tau_{1}<Y_{i t}^{*} \leq \tau_{2}, Y_{i t}=3$ if $\tau_{2}<Y_{i t}^{*} \leq \tau_{3}$, and $Y_{i t}=4$ if $Y_{i t}^{*}>\tau_{3}$, where $t_{1}, t_{2}$, and $\mathrm{t}_{3}$ are the category cutpoints. As is well known, not all the parameters $\left(\tau_{1}, \tau_{2}, \tau_{3}, \sigma^{2}, \mu_{t}, t=\right.$ $1, \ldots, T)$ of the cumulative probit model are identified, and two restrictions must be imposed to set the location and scale of the latent attitude $Y^{*}$. Typically, the restrictions $\tau_{1}=0$ and $\sigma^{2}=1$ are used, although other identifying rules may be more useful in particular applications, as herein. A graphical interpretation of the model can also be given (see fig. 1). Respondents with fewer than $\tau_{1}=-1$ units of the latent attitude $Y^{*}$ answer the NES item using response category 1 , while respondents between $\tau_{1}=-1$ and $\tau_{1}=0$ units choose response category 2 , and so on.

The usual cumulative probit model cannot be used to model opinion polarization because the variance of the latent attitude is constrained to be the same at all time points in this model. We remove this deficiency, allowing the variance of the latent attitude to depend on time (see Sobel [1998] for closely related material). In addition, we allow for the possibility that one or more of the cutpoints may change over time; this feature is demonstrated in our empirical analysis. In previous work, Terza (1985) allowed for variable cutpoints in the cumulative probit model, and McCullagh and Nelder (1989) discussed extensions of the proportional odds model where the variance is allowed to depend on independent variables. We also note that the kurtosis of any normal distribution is zero. Were we, following DiMaggio et al. (1996), to argue that declining kurtosis evidences bimodality and is therefore also necessary for establishing increasing polarization and were our model to fit the data, the null hypothesis of no increase in polarization would be accepted (even if the variance was increasing). There are two problems with such an argument. First, kurtosis does not indicate bimodality (Balanda and MacGillivray 1988). Second, in our view, bimodality does not necessarily evidence polarization; just consider the case where the modes are "very 


\section{( $Y$ = observed ordinal variable $)$}

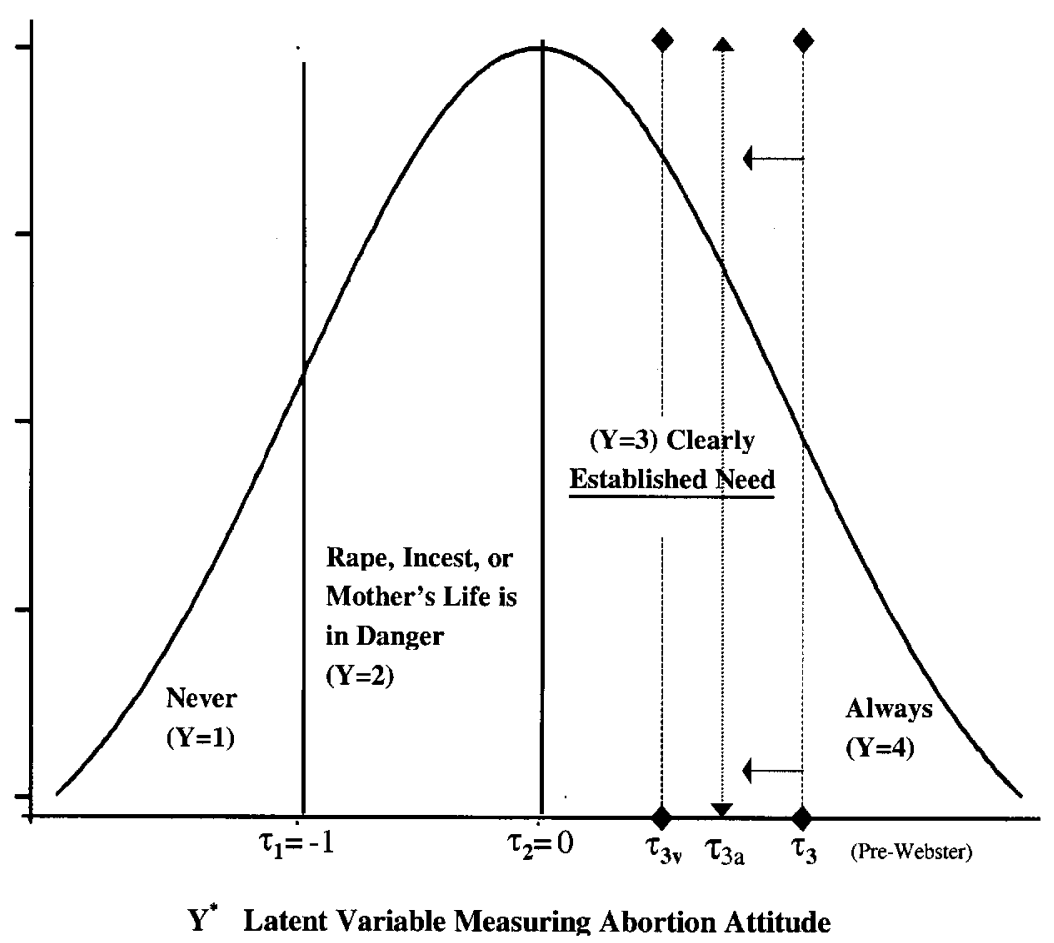

FIG. 1.-Hypothesized effect of the 1989 Webster decision on the category 3 cutpoint, $\tau_{3}$

close." However, in our view, it is intuitively clear that an increase in the variance of normally distributed random variable evidences increasing polarization.

Under our model, the parameters of substantive interest are $\mu_{t}$ (the mean at time $t$ ) and $\sigma_{t}^{2}$ (the variance at time $\left.t\right), t=1, \ldots, T$. To identify the model, we set $\tau_{1}$ and $\tau_{2}$ to zero and one, respectively (for all time periods). In a number of the models considered later, the cutpoint $\tau_{3}$ is allowed to change over time. Using these identification rules, a mean $\mu_{t}>0$ indicates that more than half the respondents at time $t$ respond using category 3 or category 4 , while fewer than half the respondents use these categories if $\mu_{t}<0$. The variances are also not identified, but fixing the cutpoints identifies the scale of the latent variable. As a result, the absolute magnitude of the variance is not meaningful herein, but the relative sizes of the variances are identified; that is, the variances can be compared over time. We test the culture wars hypothesis that polarization 


\section{Culture Wars}

is increasing over time by looking for trends in the variance of the latent variable.

The method of maximum likelihood is used to estimate the model. Under the usual types of regularity conditions (met here), the parameter estimates are consistent and asymptotically normally distributed in large samples. The computations were performed using the software package Stata (StataCorp 1997; for further details, such as the log likelihood and the first and second derivatives, see app. A).

\section{FINDINGS}

Old NES Item, 1972-80

We begin by analyzing the old NES item for the years 1972-80 (see table 2). For comparative purposes, we first note that DiMaggio et al. would have found no trend in the variance (slope $=.003$; $\mathrm{SE}=.004$ ) of the old NES question between 1972 and 1980 by inappropriately treating the data in the upper part of table 2 as interval data and regressing the series of variances on time. ${ }^{6}$ Table 5 presents model evaluation statistics for a number of the models we fit to these data (models $H_{1}-H_{5}$ ). The Bayesian information criterion, or BIC (Raftery 1995) is calculated here as $L_{2}-$ $d f \times \ln (N$ of cases). Lower levels of BIC indicate preferred models. In this article, we use $L^{2}$ to assess the model fit, and we use BIC as a secondary selection criterion.

First, we considered model $H_{1}$. In this model, the cutpoint is constant across years, but the means and variances are allowed to differ in each year. To insure that the cutpoint $\tau_{3}>0$, we parameterized the model using $\ln \left(\tau_{3}\right)$; similarly, we estimated $\ln \left(\sigma_{t}^{2}\right)$. To obtain the residual degrees of freedom for this model, note that nine parameters are estimated: one cutpoint, $\tau_{3}$, and two parameters for each of the four time points-the mean $\mu_{t}$ of the latent variable and the variance $\sigma_{t}^{2}$. In addition, at each time, the response proportions must add to one, imposing four additional constraints. Thus, the number of constraints on the 16 proportions is 13 , hence there are $3 d f$. The degrees of freedom for the models subsequently considered can be determined analogously. It is evident that $H_{1}$ fits the data adequately; the $P$-value associated with $L^{2}\left(H_{1}\right)$ is .87 . That is, our latent variable model accounts for the distribution of the observed NES item.

We now ask whether or not the variances increased over the study period, considering model $\mathrm{H}_{2}$, in which the variances (but not the means)

\footnotetext{
${ }^{6}$ In addition, for the mean series, the estimated slope is .010 ; $\mathrm{SE}=.001$; for the kurtosis series, the estimated slope is $-.015 ; \mathrm{SE}=.004 ; P=.073$.
} 


\section{American Journal of Sociology}

are equal in all years; this model also fits the data adequately $(P=.71)$ and $H_{1}$ does not fit the data better $\left(L^{2}\left[H_{2}\right]-L^{2}\left[H_{1}\right]=3 ; d f=3 ; P=\right.$ .391). Thus, we conclude (for the old NES item) that there is no polarization between 1972 and 1980 .

We also ask if there is a trend in the means between 1972 and 1980. Model $H_{3}$ is the special case of $H_{1}$ in which the means are constant between 1972 and 1980; comparing $H_{3}$ with $H_{1}\left(L^{2}\left[H_{3}\right]-L^{2}\left[H_{1}\right]=7.52 ; d f=3\right.$; $P=.057)$ suggests the means may vary over this period. Similarly, model $\mathrm{H}_{4}$ is the special case of $H_{2}$ in which the means are constant between 1972 and 1980, and comparison of $H_{4}$ with $H_{2}$ again points to variability in the means $\left(L^{2}\left[H_{4}\right]-L^{2}\left[H_{2}\right]=7.07 ; d f=3 ; P=.067\right)$. Therefore, we fit model $H_{5}$ with constant variance and a linear trend in the mean; this model fits the data almost as well as $H_{2}$, but with two fewer parameters. In addition, $H_{5}$ is clearly preferred over $H_{4}$ on the basis of the likelihood ratio test $\left(L^{2}\left[H_{4}\right]-L^{2}\left[H_{5}\right]=7.07 ; d f=1 ; P=.008\right)$; further, although the BIC statistic would suggest favoring $H_{4}$ over $H_{5}$, the difference between the two values is trivial.

New NES Item, 1980-94

Next, we model the new NES item between 1980 and 1994. If DiMaggio et al. (1996) had analyzed this series by inappropriately treating the 1980-94 data in table 2 as interval and regressing the series of means, the natural logarithm of the variances, and the kurtosis series on time, they would have concluded that there is strong evidence that attitudinal variability increased between 1980 and 1994 (slope .004; $\mathrm{SE}=.001$ ), and somewhat weaker evidence suggesting that attitudes may have liberalized over this period (slope .009; $\mathrm{SE}=.005$ ). For the kurtosis series, the slope is -.0013 with standard error $.004 .^{7}$

Table 5 presents model evaluation statistics for a number of the models we considered. Under model $M_{1}$, the means and variances are allowed to vary across survey years; however, the cutpoint $\tau_{3}$ does not vary by year. It is evident that this model does not fit the data adequately $(P<.001)$. However, before concluding that the data are incompatible with the hypothesis of a normally distributed latent attitude toward legalized abortion, we reexamine the assumption in model $M_{1}$ that the cutpoints are constant from 1980 to 1994 . It is important to note that by allowing all the cutpoints to vary freely over time, the proportions in table 2 can be reproduced exactly. That is, a model with variable cutpoints can always be obtained that will fit these data, whether or not the model correctly describes the underlying process generating the data. Consequently, we

${ }^{7}$ For the kurtosis series, the slope is $-.0013 ; \mathrm{SE}=.004$. 


\section{Culture Wars}

TABLE 5

Model Evaluation Statistics for Models of Attitudes toward LEgALIZED ABORTION

\begin{tabular}{|c|c|c|c|c|}
\hline Model & Description & $L^{2}$ & $\mathrm{BIC}$ & $d f$ \\
\hline \multicolumn{5}{|l|}{ 1972-80 data: } \\
\hline$H_{1} \ldots \ldots \ldots$ & Fit all means and variances and $\tau_{3}$ & .72 & -26.24 & 3 \\
\hline$H_{2} \ldots \ldots \ldots$ & $H_{1}$ with constant variance & 3.72 & -50.20 & 6 \\
\hline$H_{3} \ldots \ldots \ldots$ & $H_{1}$ with constant means & 8.24 & -45.68 & 6 \\
\hline$H_{4} \ldots \ldots \ldots$ & $H_{2}$ with constant means & 10.89 & -69.98 & 9 \\
\hline$H_{5} \ldots \ldots \ldots$ & $H_{2}$ with linear trend in means & 3.82 & -68.07 & 8 \\
\hline \multicolumn{5}{|l|}{ 1980-94 data: } \\
\hline$M_{1} \ldots$ & Fit all means and variances and $\tau_{3}$ & 35.28 & -31.14 & 7 \\
\hline$M_{2} \ldots \ldots \ldots$ & $M_{1}$ with $\tau_{3}$ change for $1990-94$ & 6.14 & -50.79 & 6 \\
\hline$M_{3} \ldots \ldots \ldots$ & $M_{2}$ with constant variance & 19.91 & -103.43 & 13 \\
\hline$M_{4} \ldots \ldots \ldots$ & $M_{2}$ with linear trend in $\ln$ (variance) $\tau_{3}$ & 19.58 & -94.28 & 12 \\
\hline$M_{5} \ldots \ldots \ldots$ & $M_{2}$ with different variance in 1986 & 13.16 & -99.70 & 12 \\
\hline$M_{6} \ldots \ldots \ldots$ & $M_{3}$ with constant means & 46.88 & -142.88 & 20 \\
\hline$M_{7} \ldots \ldots \ldots$ & $M_{3}$ with linear trend in means & 44.94 & -135.33 & 19 \\
\hline$M_{8} \ldots \ldots \ldots$ & $M_{6}$ with different mean in 1992 & 24.25 & -156.03 & 19 \\
\hline$M_{9} \ldots \ldots \ldots$ & $M_{1}+$ linear trend in $\ln ($ variance $)$ & 49.03 & -74.32 & 13 \\
\hline$M_{10} \ldots \ldots$ & $\begin{array}{l}M_{1}+\text { constant variance with different } \\
\text { variance in } 1986 \text { and 1990-94 }\end{array}$ & 42.72 & -71.14 & 12 \\
\hline$M_{11} \ldots$ & $\begin{array}{l}M_{2}+\text { constant means with different } \\
\quad \text { mean in } 1992\end{array}$ & 68.91 & -101.88 & 18 \\
\hline
\end{tabular}

believe that models with variable cutpoints are useful only when theoretical considerations strongly suggest their use, and we would further argue that hypotheses about cutpoint changes should be empirically testable.

To begin our reexamination, note that for categories 1 and 2 in table $\mathrm{B} 1$, the language in the NES abortion item clearly specifies the circumstances under which the law should permit abortion, but this is not the case for category 3 . Whereas the phrase "permit abortion for reasons other than rape, incest, or danger to the woman's life" demarcates response category 3 from category 2 , the circumstances that constitute the clear establishment of a need for an abortion are not fixed by the item, leaving respondents to demarcate the choice between categories 3 and 4. Significantly, the item also does not say who establishes the "need" for an abortion. Is it the woman and her doctor, or is it the state? As a result of this ambiguity, we believe that there are good reasons to suspect that changes in the public discourse on abortion rights caused this point of demarcation to change after 1989 as the result of media and activist organizations' response to the 1989 Webster decision. In July 1989, the Supreme Court, in Webster v. Reproductive Health Services, rendered the most important legal ruling on abortion since Roe v. Wade in 1973. The 
American Journal of Sociology

Webster decision ruled that restrictions on access to legal abortions were not unconstitutional, upholding a Missouri statute prohibiting the use of public facilities to perform abortion and requiring physicians to test for fetal viability after 20 weeks of pregnancy (Cook 1998).

The Webster ruling indicated that states could now attempt to restrict abortion access (Garrow 1994), and as a result, the abortion issue was thrust into the political arena as state legislatures proposed legislation restricting public funding for abortions, requiring parental consent, and testing for fetal viability (Saletan 1997). Subsequently, the Supreme Court's willingness to approve restrictive legislation in Webster was supported by the 1992 Planned Parenthood v. Casey decision, which upheld the constitutionality of restrictive legislation in Pennsylvania that required a 24-hour waiting period for abortions, parental consent, and reports on abortions performed in publicly funded institutions. Currently, 29 states have passed legislation requiring parental consent of minors' abortion decisions, 14 require a delay following state-directed counseling, 13 restrict private or public insurance coverage, 34 restrict Medicaid coverage, and 26 restrict postviability abortions. ${ }^{8}$

Although most people may not understand the intricacies of the Webster decision (see Hunter [1994] for a depiction of the public's poor understanding of Supreme Court rulings about abortion), we argue that the public discourse on abortion after Webster affected respondents' interpretation of the NES abortion item. In particular, we argue that the public discourse affected the semantics of category 3 of the NES question (see app. B), which stresses the legitimacy of abortion under the subjectively defined circumstances of "clearly established need," by associating it with increased government control over what is and is not a legitimate abortion. Even though the proposed restrictions of the Webster decision may not prohibit most women from obtaining abortions, many pro-choice organizations viewed them as a direct threat to the Roe v. Wade framework. The National Organization for Women, for example, has argued that although late-term abortions constitute only a tiny fraction of all abortions, bans on late-term and partial-birth abortions are worded ambiguously and "are intended to restrict access to all abortions." In a review of the politics of abortion rights, Saletan (1997) argued that the Webster case galvanized the pro-choice movement to reframe its defense of abortion rights. For example, the National Abortion Rights Action League

${ }^{8}$ See The Status of Major Abortion-Related Laws and Policies in the States, December 31, 2000, Alan Guttmacher Institute special report at http://www.agi-usa.org /pubs/abort_law_status.html

${ }^{9}$ Issue Report: Abortion Procedure Bans, NOW at http://www.now.org/issues /abortion/laterep.html; we viewed this document in 1999. 
(NARAL) adopted the new slogan "Who Decides-You or Them?" Saletan (1997, p. 118) argues that the value of this slogan was that it could appeal to a broader audience: "Voters with conservative instincts who shied away from the outright prohibition of immoral behavior could embrace NARAL's new message as a rejection of big government. In the minds of these voters, 'them' meant nosy, corrupt politicians and bureaucrats; 'you' meant families and communities."

Before the Webster decision, many NES respondents may have assumed that establishing the "need" for an abortion was the result of a private decision between a woman and her doctor. At the time, support for abortion in cases where the desire for an abortion was established in consultation with a doctor was high. For example, before the Webster decision, a CBS/New York Times survey in April 1989 indicated that $72 \%$ of those with opinions said that "if a woman wants to have an abortion and her doctor agrees to it," she should be allowed to have an abortion (Dionne 1989). However, the abortion discourse post-Webster encourages respondents to think that state governments will increasingly intervene in the doctor-patient relationship by placing additional restrictions on abortions that weaken the original legal framework of Roe v. Wade. In other words, who will decide when the need for an abortion has been "clearly established"- the woman and her doctor or the state? As a result, respondents may now associate the ambiguous language of the third category of the NES abortion question with increased state control over what constitutes a legitimate need for abortion. We argue that, as a result, the cutpoint separating categories 3 and 4 decreased post-Webster.

The rationale for the foregoing argument can also be seen from figure 1. Respondents whose latent attitude toward abortion $\left(Y^{*}\right)$ is less than -1 respond that the law should never permit abortion. Similarly, respondents with values of $Y^{*}$ between -1 and 0 respond that the law should permit abortion only in cases of rape, incest, or danger to the mother's life. In both of these cases, the circumstances under which abortion is permissible are clearly specified by the item, and thus there is no reason to hypothesize a change in cutpoints pre- and post-Webster. Now consider two hypothetical respondents. Suppose that while the first respondent would endorse abortion in circumstances other than rape, incest, or danger to the mother's life, he/she also favors restrictive legislation requiring fetal viability testing, parental consent, or bans on public funds for abortions. In terms of figure 1, this respondent's value of $Y^{*}$ is between 0 and $t_{3 v}$, where $t_{3 v}$ represents the dividing line between those who agree or do not agree with increased state control over what constitutes a legitimate abortion. In contrast, suppose the second respondent does not agree with these proposed restrictions, but also does not think abortion should be legal in every situation. In terms of figure 1 , this respondent's value of $Y^{*}$ is 
American Journal of Sociology

between $t_{3 v}$ and $t_{3}$. If the NES item analyzed here were administered to these two respondents prior to 1989, both will answer the item using response category 3 .

However, as argued above, the discourse on abortion rights after 1989 may cause the cutpoint $t_{3}$ to decrease because the phrase "clearly established need" is now associated with the threat of increased state control-fetal viability tests, parental consent laws, bans on public funding etc. How would this affect individual responses to the NES abortion question? After the ruling, the first respondent will still answer using response category 3 despite the shift in the third cutpoint, because he or she agrees with the proposed restrictions. In contrast, however, the second respondent is now forced to choose either category 4 , requiring him/her to endorse abortion under a wider range of circumstances than he/she would ideally want to permit, or to choose category 3 , which is now associated with increased state control in regulating a woman's "need" for an abortion. Presumably, if the second respondent's value of $Y^{*}$ is "very close" to $t_{3 v}$, he/she will choose category 3 , and if this value is "very close" to $t_{3}$, category 4 will be chosen. Thus, it is reasonable to hypothesize, as herein, that there is a point $t_{3 a}$, between $t_{3 v}$ and $t_{3}$ such that respondents with more than $t_{3 a}$ units of $Y^{*}$ choose category 4, while respondents with between 0 and $t_{3 a}$ units of $Y^{*}$ choose category 3. $t_{3 a}$ is not the same as the hypothetical cutpoint $t_{3 v}$ because $t_{3 v}$ represents the dividing line between respondents based upon the hypothetical division of category 3 into two distinct categories (resulting in a total of 5 categories). In contrast, $t_{3 a}$ represents the shift of the cutpoint dividing categories 3 and 4 in the case where there are only 4 categories. In other words, after the Webster decision, the third cutpoint shifts to the left from $t_{3}$ to $t_{3 a}$.

In light of this hypothesized effect of the abortion discourse after 1989 on the NES item, we modified model $M_{1}$, allowing the cutpoint separating categories 3 and 4 to change in 1990 and subsequent years. The resulting model $\left(M_{2}\right)$ fits the data very well $(P=.41)$, and the estimated cutpoints (standard errors in parentheses) for the first and second time periods are, respectively, .495 and .364 , consistent with our hypothesis that the cutpoint is smaller in 1990 and thereafter. To test whether or not there is a significant difference between the two cutpoints, recall that we estimated the natural logarithms of these quantities; on this scale, the difference between the two maximum-likelihood estimates is $\ln \left(\hat{\tau}_{3}\right)-\ln \left(\hat{\tau}_{3 a}\right)=.305$ $(\mathrm{SE}=.057)$. Hence a one-tailed test of the null hypothesis that the cutpoints are identical versus the alternative that the second is smaller leads to rejecting the null in favor of the alternative at the .001 level.

We now ask whether or not the variance of abortion attitudes increased over the period 1980-94. To address this issue, we consider model $M_{3}$, in which the variance is constant and the means are unrestricted. This model 
Culture Wars

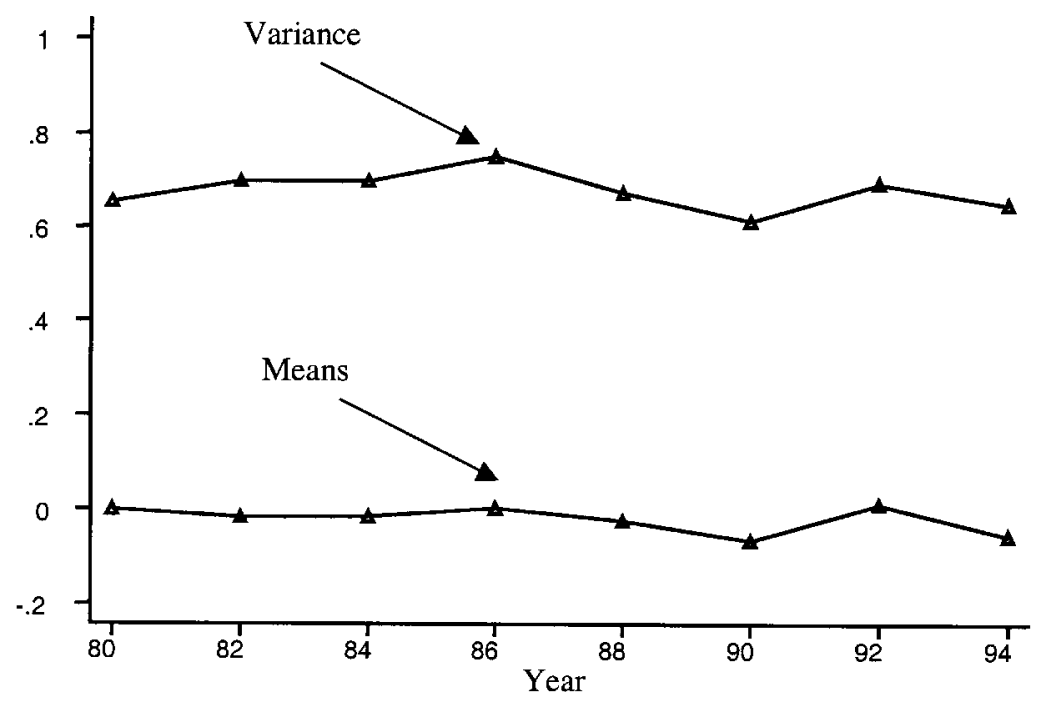

FIG. 2.-Trends in the mean and variance of abortion attitudes 1980-94 under model $M_{2}$

also fits the data adequately $(P>.05)$, consistent with the conclusion of no trend in the variance. However, the conditional likelihood ratio test of $M_{3}$ versus $M_{2}\left(L^{2}\left[M_{3}\right]-L^{2}\left[M_{2}\right]=13.77 ; d f=7\right)$ has $P$ value of .055 , suggesting possible heterogeneity in the variation across years. DiMaggio et al. (1996) fit a linear model to the variance estimates in their data, claiming the variance increases over time. In model $M_{4}$, the logarithms of the variances follow a linear model: $\ln \left(\sigma_{t}^{2}\right)=\alpha+\beta(t-1980)$. From table 5 it is clear that the data offer no grounds for preferring $M_{4}$ to $M_{3}$, as there is almost no improvement in $L^{2}$ at the cost of one degree of freedom $\left(L^{2}\left[M_{3}\right]-L^{2}\left[M_{4}\right]=0.33 ; d f=1\right)$; that is, the null hypothesis of no linear trend in the variance is not rejected.]

Figure 2 plots the estimated means and variances under model $M_{2}$ against time; recall that because these parameters are identified only up to location and scale, only comparisons amongst the means and the variances are of interest. The estimated $\sigma_{t}^{2}$ fluctuate irregularly, consistent with the hypothesis that the variation does not increase between 1980 and 1994. However, although there is no obvious trend in these estimates, it also appears that the variance may be larger in 1986 or 1992 than in the other years; similarly, the variance may be smaller in 1990 than in the other years. Ex post facto, this suggests considering models where the variance may be smaller (larger) in a given year than in other years, testing such models against $M_{3}$. There are eight such models, each adding a single parameter to $M_{2}$. If we had a priori substantive reasons for focusing on 


\section{American Journal of Sociology}

pre specified years, then it would be legitimate to estimate a subset of these models, but as this is not the case, all eight models should be considered, which raises the issue of overfitting the data. Therefore, to test the null hypothesis of constant variation against the alternative of nonconstant variance, we test each model against $M_{3}$ using a significance level of .05/8 (Miller 1981). Of the eight models considered, the best fitting $\left(M_{5}\right)$ allows the variance to differ in 1986. However, the likelihood-ratio test of $M_{5}$ versus $M_{3}$ is not significant at the $.05 / 8$ level, and the BIC statistic (Raftery 1995) suggests that $M_{3}$ should be preferred to $M_{5}$.

To this point, the results suggest selecting either $M_{2}$ or $M_{3}$ as the preferred model; importantly, neither of these suggests that attitudes toward legalized abortion have polarized over the period 1980-94. Of course, many models other than those considered here are nested between $M_{2}$ and $M_{3}$. However, because we think it unlikely that the conclusions about polarization one would draw from $M_{2}$ or $M_{3}$ would change by choosing such a model, we limit our selection to these two models, choosing $M_{3}$ over $M_{2}$ on the basis of the BIC statistic.

Having established that the variance did not increase between 1980 and 1994, we now turn attention to modeling the means, using $M_{3}$ as a baseline model. We first compare model $M_{6}$, in which the means are constant, with model $M_{3}$; the conditional likelihood-ratio test $\left(L^{2}\left[M_{6}\right]-\right.$ $\left.L^{2}\left[M_{3}\right]=26.97 ; d f=7 ; P<.001\right)$ suggests some heterogeneity. Following DiMaggio et al. (1996), we model the means as a linear function of time in model $M_{7}$; the model does not fit the data as well as $M_{3}\left(L^{2}\left[M_{7}\right]-\right.$ $\left.L^{2}\left[M_{3}\right]=25.03 ; d f=6 ; P<.001\right)$, and the fit is virtually identical to that of $M_{6}$.

Figure 2 suggests the mean is larger in 1992 than in the other years; therefore model $M_{8}$ allows the mean to differ in this year. Note that model $M_{3}$ does not fit better than $M_{8}\left(L^{2}\left[M_{8}\right]-L^{2}\left[M_{3}\right]=4.34 ; d f=7 ; P=.74\right)$ and that if the BIC statistic is used to select between these models, $M_{8}$ is preferred. Note also that $M_{8}$ is preferred to the more parsimonious $M_{6}$ on these grounds. Finally, although $M_{6}$ does not fit the data unconditionally at the .05 level, the difference $L^{2}\left(M_{8}\right)-L^{2}\left(M_{6}\right)=22.63$ on one degree of freedom suggests a large improvement, and even though the contribution from $M_{6}$ does not have a chi-square distribution, the chi-square distribution for differences between $L^{2}$ values is often approximately valid in such cases; assuming the validity of this approximation, the $P$ value is less than .001 , substantially less than $.05 / 8$.

Our results thus lead to selecting model $M_{8}$ to describe the data; table 6 reports the estimated variance, the two estimated means $\left(\mu_{1}\right.$ is the mean for all years except 1992), and the two estimated cutpoints. The estimated cutpoint prior to the Webster decision is .491, while the estimated cutpoint for 1990 and later is .369. As before, the ordering is consistent with our 


\section{Culture Wars}

TABLE 6

Descriptive Statistics For $M_{8}$

\begin{tabular}{lcc}
\hline \hline Parameter & Estimate & $\mathrm{SE}$ \\
\hline $\ln \left(t_{3}\right)^{*} \ldots \ldots$ & -.711 & .027 \\
$\ln \left(t_{3 a}\right)^{\dagger} \ldots$. & -.995 & .041 \\
$M_{1} \ldots \ldots$. & .151 & .012 \\
$\mathrm{~m}_{1992} \ldots \ldots$ & .325 & .036 \\
$\ln \left(s^{2}\right)^{\ddagger} \ldots$ & .029 & .027 \\
\hline$* t_{3}=\exp (-.711)=.491$. & \\
$t_{3 a}=\exp (-.995)=.369$. & \\
${ }^{\dagger} s^{2}=\exp (.029)=1.03$. &
\end{tabular}

a priori expectations, and the differences between the estimates is significant, on the basis of a 1 tailed test, at the .001 level of significance. Although we cannot explain why attitudes are more favorable to legalized abortion in 1992 than in the other years, the evidence does not support the hypothesis that Americans are becoming more favorable to legalized abortion over the study period. Finally, recalling that the variance is constant and that the kurtosis is also constant because the latent attitude is normally distributed, the data offer no support for the hypothesis of increasing polarization.

Our substantive conclusions about the 1980-94 period are obtained using models for ordinal variables in which the cutpoint separating the third and fourth categories of the NES item changes after the Webster decision. The models fit the data well, whereas models that do not allow this cutpoint change do not fit the data. We have also given good reasons to expect such a change and correctly specified the direction of this change. Further, the empirical impact of this change seems reasonable; using the preferred model $M_{8}$, in 1990, $14.4 \%$ of the respondents are predicted to fall in category $3,41.5 \%$ in category 4 . If the pre-Webster cutpoint were used instead, $4.6 \%$ of the respondents would be reallocated from category 4 to category 3 . In addition, the best-fitting model with a cutpoint change occurs at the time point we identified a priori; no model with a cutpoint change in another year fits the data at the .05 level. For the model with a cutpoint shift in 1982, $L^{2}=35.3(d f=6 ; P<.001)$. For $1984 L^{2}=$ $33.4(d f=6 ; P<.001)$, and for $1986, L^{2}=23.3(d f=6 ; P<.001)$. For the model with a shift in $1988, L^{2}=11.89(d f=6 ; P=.06)$; the near fit of this model is explained by its similarity to $M_{2}$. For the model with a cutpoint shift in $1992, L^{2}=28.0(d f=6 ; P<.001)$; for the model with a shift in $1994, L^{2}=31.3(d f=6 ; P<.001)$. Despite the strong justifications we have given, a critic could always attack our conclusions, arguing that the assumptions used to construct the statistical model are faulty or that our use of $L^{2}$ to measure both goodness of fit and aid in 


\section{American Journal of Sociology}

the model selection process is misguided; for example, obviously one could argue that instead of a cutpoint change, the latent variable follows some unspecified distribution other than the normal after 1988. Similarly, as the model parameters are not identified (see eq. 2 in app. A), conclusions about the variability of the series can depend on the parameterization of the model. Although we believe our parameterization offers the most plausible representation of the behavioral process pre- and post-Webster respondents might have employed in responding to the new NES item, a critic could always reject our line of argument. We cannot address all these potential criticisms, nor can we handle all of these within the modeling framework proposed herein, and even were we to construct a more general framework, untestable assumptions used in the model construction process would still be a target for criticism.

Although we believe our analysis is compelling, it is simply less definitive than would be the case were the NES item measured at the interval or ratio level. For the sake of completeness and so that the reader may judge all the evidence for him or herself, we now report the findings we would have obtained had we confined attention to models without a cutpoint change. In that vein, recall that under model $M_{1}$, the means and variances were allowed to vary over the survey years, but the cutpoint was the same in all years; we rejected this model because it did not fit the data at any reasonable significance level, using $L^{2}$. However, given the large sample size, one might try to argue that model $M_{1}$ provides a reasonable description of the data (this is not an argument we would endorse, but it is not an uncommon type of argument). Were we to proceed along such lines, a natural model to consider next is $M_{9}$; this is a special case of model $M_{1}$ in which the logarithms of the variances change linearly over time. Were we to accept the results from model $M_{9}$ at face value, the trend coefficient is significantly different from zero at the .05 level (.023; SE $=.005)$. However, if we use the conditional-likelihood test, $M_{9}$ is inferior to $M_{1}\left(L^{2}\left[M_{9}\right]-L^{2}\left[M_{1}\right]=13.75 ; d f=6 ; P=.03\right)$, which suggests that a linear trend does not adequately capture the temporal change in attitudinal variability. In addition, the variances were modeled using polynomials up to order 3 with no improvement. Next, inspection of the variances estimated under model $M_{1}$ suggested model $M_{10}$, in which there are three distinct variances ( $\sigma_{1}^{2}$ for 1980,1984 , and 1988; $\sigma_{2}^{2}$ for 1986; and $\sigma_{3}^{2}$ for 1990,1992 , and 1994). Under this model, $\sigma_{3}^{2}$ is greater than $\sigma_{1}^{2}$ (using the .05 level), suggesting (with the exception of 1986) that polarization is higher in the 1990s than the 1980s. A similar pattern shows up in model $M_{11}$; this model is obtained from $M_{10}$ by constraining the means (except for the mean in 1992) to be identical.

The ad hoc models $M_{10}$ and $M_{11}$ do not support the hypothesis that polarization is monotonically increasing over time, but they do suggest 
ex-post facto that the variability of abortion attitudes is greater in the 1990s than in the 1980s (save 1986). However, none of the models above come close to fitting the data at conventionally used significance levels, and if the BIC is used as a model selection criterion, model $M_{8}$, in which the variability of abortion attitudes is constant, is still clearly preferred to any of those just considered.

\section{DISCUSSION}

Sociologists and journalists have argued that recent "cultural wars" over politicized moral issues have resulted in rising levels of attitude polarization and social conflict. According to these writers, the level of polarization and fragmentation among the public as a whole is so irreconcilable that democratic compromise on these issues is increasingly difficult, posing a strong threat to the viability of American political institutions. The controversy over abortion exemplifies the concerns of these writers, who often use this as the leading example with which to make their case. Typically, however, no empirical evidence of increased attitudinal polarization is presented; rather, the purported increase is indirectly inferred from the outcomes with which such polarization is (in theory) associated. In an important contribution, DiMaggio et al. (1996) examined temporal trends in public opinion data to assess this thesis, finding little evidence of increasing polarization, with opinions about abortion constituting the major exception. In general, however, their conclusions may be misleading because they analyze nominal and ordinal opinion items using methods designed for interval measurements. In particular, DiMaggio et al. inappropriately analyze both the GSS data and the NES data on abortion.

We develop new methods for studying opinion polarization using ordinal data. In our model, a generalization of the cumulative probit model, the latent attitude toward abortion follows a normal distribution at each time point. In contrast to DiMaggio et al., our results do not suggest an increase in polarization. Interestingly, with respect to abortion, one of the most controversial public issues, our results suggest support for the more general conclusion in DiMaggio et al.: public opinion has not polarized much over the last 25 years. Nonetheless, two caveats are in order. First, because our model is not appropriate for analyzing the GSS data on abortion, our conclusions are based solely on the analysis of the NES item; it is conceivable that an appropriate analysis of the GSS data would find evidence of increasing polarization. Second, the latent variable in our analysis is assumed to follow a normal distribution, and in our analysis of the 1980-94 data, we allowed the cutpoint separating categories 3 and 4 to change after 1988. Although we offered both theoretical and empirical 
American Journal of Sociology

support for this analysis, because we are using ordinal data and must therefore make assumptions that cannot be fully tested, our analysis will always be open to criticism by those who want to maintain that polarization in abortion attitudes has been increasing. However, at this point, we believe that our analyses are strong enough to place the burden of proof upon the would-be critic. In future work on this topic, we intend to extend our model to include distributions other than the normal, and we intend to develop appropriate methods for studying polarization when multiple items are used to measure the underlying attitude (as is the case in the GSS).

Do the culture wars exist? Proponents of the culture wars hypothesis claim that America is becoming increasingly divided over politicized moral issues. Abortion is an issue where the debate is particularly divisive. Hunter (1991, 1996), for instance, argues that because of the sound-bite society we live in, special interest groups and political organizations attempt to inflame public opinion about abortion and undermine their opponents rather than participate in genuine debate. This contributes to a "hollowing out" of the possibility of achieving consensus and raises the specter of violence as a means of imposing one's beliefs upon others. However, our results show that there is no evidence that Americans as a whole became more divided over abortion rights between 1972 and 1980 nor between 1980 and 1994. In the lag between submission and publication of this article, new NES data have become available, and we have extended the 1980-94 analysis to 2000. In addition to models analogous to those considered in this paper, we designed new models to ascertain whether polarization had increased during the 1990s. Our results indicate no increase in polarization in abortion attitudes between 1980 and 2000 (complete results available from authors on request). While the contemporary culture wars have been portrayed as a threat to democratic institutions, our evidence suggests that, at least with respect to the debate over abortion rights, the American public is no more divided now than in the past.

\section{APPENDIX A}

\section{Estimation of the Model}

Our model extends the usual cumulative probit model to allow for heteroscedasticity and variable cutpoints. Let $Y$ denote an observed ordinal variable with response categories $j=1, \ldots, J$, let $Y^{*}$ denote a metrical latent variable, and let $\mathbf{X}$ denote a vector of covariates. Under the model herein, the distribution of $Y^{*}$, given $\mathbf{X}=\mathbf{x}$ (denoted $\phi[y * \mid \mathbf{x}]$ ) is normal, with mean $\mu(\mathbf{x} ; \theta)$ and standard deviation $\sigma(\mathbf{x} ; \theta)$, where $\theta$ is a vector of 


\section{Culture Wars}

parameters to be estimated. The observed variable $Y$ is a quantized version of the latent variable $Y^{*}$; for each level $\mathbf{x}$ of the covariates, $Y=1$ if and only if $Y^{*}<\tau_{1}(\mathbf{x} ; \theta), Y=2$ if and only if $\tau_{1}(\mathbf{x} ; \theta)<Y^{*} \leq \tau_{2}(\mathbf{x} ; \theta), \ldots$ ,$\ldots$, and $Y=J$ if and only if $Y^{*}>\tau_{J-1}(\mathbf{x} ; \theta)$. It follows that

$$
\operatorname{prob}(Y=j \mid \mathbf{X}=\mathbf{x})=\Phi\left[\frac{\tau_{j}(\mathbf{x} ; \theta)-\mu(\mathbf{x} ; \theta)}{\sigma(\mathbf{x} ; \theta)}\right]-\Phi\left[\frac{\tau_{j-1}(\mathbf{x} ; \theta)-\mu(\mathbf{x} ; \theta)}{\sigma(\mathbf{x} ; \theta)}\right],
$$

where $\Phi$ denotes the distribution function of the standard normal distribution, and for all $\mathbf{x}, \tau_{0}(\mathbf{x} ; \theta)=-\infty, \tau_{J}(\mathbf{x} ; \theta)=\infty$. As

$$
z_{j}(\mathbf{x} ; \theta)=\frac{\tau_{j}(\mathbf{x} ; \theta)-\mu(\mathbf{x} ; \theta)}{\sigma(\mathbf{x} ; \theta)}=\frac{\kappa_{\mathbf{x}}\left[\tau_{j}(\mathbf{x} ; \theta)+\lambda_{\mathbf{x}}\right]-\left[\mu(\mathbf{x} ; \theta)+\lambda_{\mathbf{x}}\right]}{\kappa_{\mathbf{x}} \sigma(\mathbf{x} ; \theta)},
$$

it is evident that at each level of the covariates, the parameters $\mu(\mathbf{x} ; \theta)$ and $\sigma(\mathbf{x} ; \theta)$ are identified only up to location and scale. In the usual cumulative probit model, it is assumed that neither the cutpoints $\tau_{j}(\mathbf{x} ; \theta)$ nor the variance $\sigma^{2}(\mathbf{x} ; \theta)$ depends on $\mathbf{x}$. Therefore, only two restrictions need be imposed to identify the model; the restrictions $\tau_{1}(\mathbf{x} ; \theta)=\tau_{1}=0$ and $\sigma^{2}(\mathbf{x} ; \theta)=\sigma^{2}=1$ are conventionally used, though other restrictions could be used and might be more desirable in certain types of applications.

We now briefly discuss maximum-likelihood estimation of the extended model. We assume the parameter vector $\theta$ is identified (as is the case for the models considered in this paper).

Let $\left(\mathbf{x}_{i}, y_{i}\right), i=1, \ldots, n$, denote the observed data (hereafter $D$ ), and, for $j=1, \ldots, J$, let $y_{i j}=1$ if $y_{i}=j$, otherwise. The log likelihood is

$$
\begin{aligned}
\ln L(\theta ; D) & =\sum_{i=1}^{n} \sum_{j=1}^{J} y_{i j} n \ln \operatorname{prob}\left(Y=j \mid \mathbf{X}_{i}=\mathbf{x}\right) \ln \\
& =\sum_{i=1}^{n} \sum_{j=1}^{J} y_{i j} \ln \left\{\Phi\left[z_{j}(\mathbf{x} ; \theta)\right]-\Phi\left[z_{j-1}(\mathbf{x} ; \theta)\right]\right\},
\end{aligned}
$$

and this is to be maximized with respect to $\theta$.

Under the usual types of regularity conditions (see, e.g., Amemiya 1985), a maximum-likelihood estimator $\hat{\theta}$ of $\theta$ is a solution of the likelihood equations:

$$
\begin{aligned}
\frac{\partial \ln L(\theta ; D)}{\partial \theta} & =\sum_{i=1}^{n} \sum_{j=1}^{J} y_{i j}\left[\operatorname{prob}\left(Y_{i}=j \mid \mathbf{X}_{i}=\mathbf{x}_{i}\right)\right] \frac{\partial \operatorname{prob}\left(Y_{i}=j \mid \mathbf{X}_{i}=\mathbf{x}_{i}\right)}{\partial \theta} \\
& =\sum_{i=1}^{n} \sum_{j=1}^{J} \frac{y_{i j} \phi\left[z_{j}\left(\mathbf{x}_{i} ; \theta\right)\right] \partial \phi\left[z_{j}\left(\mathbf{x}_{i} ; \theta\right)\right] / \partial \theta-\phi\left[z_{j-1}\left(\mathbf{x}_{i} ; \theta\right)\right] \partial \phi\left[z_{j-1}\left(\mathbf{x}_{i} ; \theta\right)\right] / \partial \theta}{\Phi\left[z_{j}\left(\mathbf{x}_{i} ; \theta\right)\right]-\Phi\left[z_{j-1}\left(\mathbf{x}_{i} ; \theta\right)\right]}=0 .
\end{aligned}
$$




\section{American Journal of Sociology}

In large samples, $\hat{\theta}$ is approximately normally distributed with mean $\theta$ and covariance matrix $n^{-1} \sum(\theta)$. The matrix $\sum(\theta)$ can be estimated consistently by $\left\{(1 / n) \times\left[\partial^{2} \ln L(\theta ; D)\right] /\left(\partial \theta \partial \theta^{\prime}\right)\right\}-1$; for the model here,

$$
\begin{aligned}
{\left[\frac{\partial^{2} \ln L(\theta ; D)}{\partial \theta \partial \theta^{\prime}}\right]=} & \sum_{i=1}^{n} \sum_{j=1}^{J} y_{i j}\left[\left(\operatorname{prob}\left(Y_{i}=j \mid \mathbf{X}_{i}=\mathbf{x}_{i}\right)\right]^{-1} \frac{\partial^{2} \operatorname{prob}\left(Y_{i}=j \mid \mathbf{X}_{i}=\mathbf{x}_{i}\right)}{\partial \theta \partial \theta^{\prime}}\right. \\
& -\left[\operatorname{prob}\left(Y_{i}=j \mid \mathbf{X}_{i}=\mathbf{x}_{i}\right)\right]^{-2} \\
& \frac{\partial \operatorname{prob}\left(Y_{i}=j \mid \mathbf{X}_{i}=\mathbf{x}_{i}\right)}{\partial \theta} \frac{\partial\left[\operatorname{prob}\left(Y_{i}=j \mid \mathbf{X}_{i}=\mathbf{x}_{i}\right)\right]}{\partial \theta^{\prime}}
\end{aligned}
$$

with

$$
\begin{aligned}
\frac{\partial^{2} \operatorname{prob}\left(Y_{i}=j \mid X_{i}=\mathbf{x}_{i}\right)}{\partial \theta \partial \theta^{\prime}}= & \frac{\partial \phi\left[z_{j}\left(\mathbf{x}_{i} ; \theta\right)\right]}{\partial \theta} \frac{\partial \phi\left[z_{j}\left(\mathbf{x}_{i} ; \theta\right)\right]}{\partial \theta^{\prime}} \\
& +\phi\left[z_{j}\left(\mathbf{x}_{i} ; \theta\right)\right] \frac{\partial^{2} \phi\left[z_{j}\left(\mathbf{x}_{i} ; \theta\right)\right]}{\partial \theta \partial \theta^{\prime}} \\
& -\frac{\partial \phi\left[z_{j-1}\left(\mathbf{x}_{i} ; \theta\right)\right]}{\partial \theta} \frac{\partial \phi\left[z_{j-1}\left(\mathbf{x}_{i} ; \theta\right)\right]}{\partial \theta^{\prime}} \\
& -\phi\left[z_{j-1}\left(\mathbf{x}_{i} ; \theta\right)\right] \frac{\partial^{2} \phi\left[z_{j-1}\left(\mathbf{x}_{i} ; \theta\right)\right]}{\partial \theta \partial \theta^{\prime}}
\end{aligned}
$$

To estimate the parameters and their standard errors, a modified NewtonRaphson algorithm using numerical derivatives for (A4) and (A5), implemented in the Stata package, was used. For further details, see the description of the linear form routine in the section on maximumlikelihood estimation in the Stata reference manual (StataCorp 1997). The code used to implement the model in Stata is available online from the authors.

Because our covariate $(X)$ is discrete, we (in order to avoid computing the $\log$ likelihood for each individual observation) maximized the equivalent grouped data log likelihood:

$$
\ln L(\theta ; D)=\sum_{t=1}^{T} \sum_{j=1}^{J} n_{t j} \ln \left[\operatorname{prob}\left(Y_{i}=j \mid \mathbf{X}_{i}=t\right)\right],
$$

where $n_{t j}$ denotes number of observations at time $t$ in category $j$ of $Y$. 


\section{Culture Wars}

\section{APPENDIX B}

\section{Abortion Questionnaire}

There has been some discussion about abortion during recent years. Which one of the opinions on this page best agrees with your view? You can just tell me the number of the opinion you choose.

TABLE B1

COMPARISON OF NEW AND Old ITEMS

\begin{tabular}{|c|c|c|}
\hline \multirow[b]{2}{*}{ CATEGORY } & \multicolumn{2}{|c|}{ OPINION/QUESTION } \\
\hline & $1972-80$ & 1980-94 \\
\hline $1 \ldots$ & Abortion should never be permitted. & $\begin{array}{l}\text { By law, abortion should never } \\
\text { be permitted. }\end{array}$ \\
\hline 2 & $\begin{array}{l}\text { Abortion should be permitted only } \\
\text { if the life and health of the } \\
\text { woman is in danger. }\end{array}$ & $\begin{array}{l}\text { The law should permit abor- } \\
\text { tion only in case of rape, in- } \\
\text { cest, or when the woman's } \\
\text { life is in danger. }\end{array}$ \\
\hline $3 \ldots \ldots \ldots$ & $\begin{array}{l}\text { Abortion should be permitted if, } \\
\text { due to personal reasons, the } \\
\text { woman would have difficulty in } \\
\text { caring for the child. }\end{array}$ & $\begin{array}{l}\text { The law should permit abor- } \\
\text { tion for reasons other than } \\
\text { rape, incest, or danger to } \\
\text { the woman's life, but only } \\
\text { after the need for the abor- } \\
\text { tion has been clearly } \\
\text { established. }\end{array}$ \\
\hline $4 \ldots \ldots \ldots$ & $\begin{array}{l}\text { Abortion should never be forbidden, } \\
\text { since one should not require a } \\
\text { woman to have a child she } \\
\text { doesn't want. }\end{array}$ & $\begin{array}{l}\text { By law, a woman should al- } \\
\text { ways be able to obtain an } \\
\text { abortion as a matter of per- } \\
\text { sonal choice. }\end{array}$ \\
\hline
\end{tabular}

\section{REFERENCES}

Adamek, Raymond. 1994. "Public Opinion and Roe v. Wade: Measurement Difficulties: The Polls-a Review." Public Opinion Quarterly 58:409-18.

Agresti, Alan. 1990. Categorical Data Analysis. New York: Wiley.

Amemiya, T. 1985. Advanced Econometrics. Cambridge, Mass.: Harvard University Press

Balanda, Kevin P., and H. L. MacGillivray 1988. "Kurtosis: A Critical Review." American Statistician 42:111-19.

Bennett, William J. 1992. The De-Valuing of America: The Fight for Our Culture and Our Children. New York: Summit Books.

Blake, Judith, and Jorge del Pinal. 1981. "Negativism, Equivocation, and Wobbly Assent: Public 'Support' for the Pro-Choice Platform on Abortion." Demography 18: 309-20.

Bloom, James D. 1992. Left Letters: The Culture Wars of Mike Gold and Joseph Freeman. New York: Columbia University Press.

Bolton, Richard, ed. 1992. Culture Wars: Documents from the Recent Controversies in the Arts. New York: New Press. 


\section{American Journal of Sociology}

Brint, Steven. 1992. "What If They Gave a War . . . ?" Contemporary Sociology 21 (4): 438-40.

Bumpass, Larry L. 1997. "The Measurement of Public Opinion on Abortion: The Effects of Survey Design." Family Planning Perspectives 29 (4): 177-80.

Claire, Miriam. 1995. The Abortion Dilemma. New York: Plenum Press.

Cook, Elizabeth, Ted Jelen, and Clyde Wilcox. 1992. Between Two Absolutes: Public Opinion and the Politics of Abortion. Boulder, Colo.: Westview Press.

Cook, Kimberly J. 1998. Divided Passions: Public Opinions on Abortion and the Death Penalty. Boston: Northeastern University Press.

Davis, Nancy, and Robert Robinson. 1996. "Are the Rumors of War Exaggerated? Religious Orthodoxy and Moral Progressivism in America." American Journal of Sociology 102:756-87.

de Boer, Connie. 1977. "The Polls: Abortion." Public Opinion Quarterly 41:553-64.

Dillon, Michele. 1996. "The American Abortion Debate." Pp. 115-32 in The American Culture Wars, edited by J. L. Nolan, Jr. Charlottesville: University Press of Virginia.

Dionne, E. J., Jr. 1989. "Poll on Abortion Finds the Nation Is Sharply Divided." New York Times, April 26, pp. 1, 13.

DiMaggio, Paul, John Evans, and Bethany Bryson. 1996. "Have Americans' Social Attitudes Become More Polarized?" American Journal of Sociology 102:690-755.

Gallup Poll News Service. 1979. The Gallup Poll: Public Opinion 1978. Wilmington, Del.: Scholarly Resources.

1998. Opinion Poll. January 16-18. (MRDF) Gallup Poll News Service.

Garrow, David J. 1994. Liberty and Sexuality. New York: Macmillan.

Gates, Henry Louis, Jr. 1992. Loose Canons: Notes on the Culture Wars. New York: Oxford Universtiy Press.

Gillespie, Michael, Elisabeth Vergert, and Johannes Kingma. 1987. "Secular Trends in Abortion Attitudes." Journal of Psychology 122:323-41.

Ginsburg, Faye. 1989. Contested Lives: The Abortion Debate in an American Community. Berkeley and Los Angeles: University of California Press.

Gitlin, Todd. 1995. The Twilight of Common Dreams: Why America Is Wracked by Culture Wars. New York: Metropolitan Books.

Glenn, Norval D. 1987. "Social Trends in the United States: Evidence from Sample Surveys.” Public Opinion Quarterly 51:S109-S126.

Granberg, Donald, and Beth Wellman Granberg. 1980. "Abortion Attitudes, 1965-1980: Trends and Determinants." Family Planning Perspectives 12:250-61.

Hall, Mimi. 1998. "Polls: Shift in Support for Abortion." USA Today, January 21, p. $1 \mathrm{~A}$.

Hartman, Geoffrey H. 1991. Minor Prophecies: The Literary Essay in the Culture Wars. Cambridge, Mass.: Harvard University Press.

Hochschild, Jennifer. 1995. Facing Up to the American Dream. Princeton, N.J.: Princeton University Press.

Hunter, James D. 1991. Culture Wars. New York: Basic Books

—. 1993. "Covering the Culture War: Before the Shooting Begins." Columbia Journalism Review, July/August, pp. 29-32.

1994. Before the Shooting Begins. New York: Free Press.

. 1996. "Reflections on the Culture Wars Hypothesis." Pp. 243-56 in The American Culture Wars, edited by J. L. Nolan, Jr. Charlottesville: University Press of Virginia.

Jacoby, Russell. 1994. Dogmatic Wisdom: How the Culture Wars Divert Education and Distract America. New York: Doubleday.

Jaffe, Frederick S., Barbara L. Lindheim, and Philip R. Lee. 1981 Abortion Politics. New York: McGraw-Hill.

Luker, Kristin. 1984. Abortion and the Politics of Motherhood. Berkeley and Los Angeles: University of California Press. 


\section{Culture Wars}

McCullagh, Peter, and John A. Nelder. 1989. Generalized Linear Models. New York: Chapman and Hall.

Miller, Rupert G., Jr. 1981. Simultaneous Statistical Inference, 2d ed. New York: Springer-Verlag.

Nolan, James L., Jr., ed. 1996. The American Culture Wars. Charlottesville: University Press of Virginia.

O'Connor, Karen. 1996. No Neutral Ground? Boulder, Colo.: Westview Press.

Page, Benjamin I., and Robert Y. Shapiro. 1992. The Rational Public. Chicago: University of Chicago Press.

Raftery, Adrian E. 1995. "Bayesian Model Selection in Social Research" (with discussion). Sociological Methodology 25:111-95.

Risen, James, and Judy L. Thomas. 1998. Wrath of Angels: The American Abortion War. New York: Basic Books.

Saletan, William. 1997. "Electoral Politics and Abortion: Narrowing the Message." Pp. 111-23 in Abortion Wars, edited by Rickie Solinger. Berkeley and Los Angeles: University of California Press.

Shor, Ira. 1986. Culture Wars: School and Society in the Conservative Restoration, 1969-1984. Boston: Routledge.

Sobel, Michael E. 1998. "Some Log-Linear and Log-Nonlinear Models for Ordinal Scales with Midpoints, with an Application to Public Opinion Data.” Sociological Methodology 28:263-92.

StataCorp. 1997. Stata Statistical Software: Release 5.0. College Station, Tex.: Stata Corporation.

Terza, J. V. 1985. "Ordinal Probit: A Generalization." Communications in Statistics (Theory and Methods) 14:1-11.

Tribe, Laurence H. 1990. Abortion: The Clash of Absolutes. New York: W. W. Norton. Wuthnow, Robert. 1996. Christianity and Civil Society. Valley Forge, Pa.: Trinity Press International. 